\title{
Problemas ambientais, saúde coletiva e ciências sociais
}

\author{
Environmental problems, collective health \\ and social sciences
}

Carlos M achado de Freitas 1

1 Centro de Estudos da Saúde do Trabalhador e Ecologia Humana, Escola N acional de Saúde Pública, Fiocruz. Av. Leopoldo Bulhões 1480, M anguinhos, 21041-210, Rio de Janeiro RJ. carlosmf@ensp.fiocruz.br
Abstract Environmental problems relative to health conditions have been always present in the speeches and practises about health. During 19 century, however, the wide and intense impacts of industrialization and urbanization over sanitary conditions contribute to a comprehension of these problems as result of social and political processes. With the advent of microbi ol ogi cal paradigm the strategies for these problems solutions were reduced to sanitation and vector control and the social and political dimensions become marginal and peripheric. Thenotion of environmental and health problems was enlarged and the social and political dimensions were retaking only during the 70 s with the emergence of environmental movement and of social medicine in Latin-America. In spite of these advances, data analyses about researches groups, thesis and di ssertations production and articles published in scientific journals reveal the collective health have a marginal rolein the research about environmental problems and the research and scientific production of social science is too few. The present situation requires quantitative and qualitative advances in collective health research and scientific production, especially in the field of social science and health.

Key words Environmental problems, Social science, Collective health
Resumo Problemasambientais e sua interface com a saúde estão sempre presentes nos discursos e práti cas sanitárias. Em meados do século 19, com os intensos impactos do processo de industrialização e urbanização sobre as condições sanitárias e de saúde, esses problemas são vistos como resultados de processos políticos e sociais. M as com o paradigma mi crobiano essa relação foi reduzida aos problemas de saneamento e a controle de vetores. A dimensão social e política passa a ocupar lugar marginal e periférico. Para os movimentos ambientalistas e a medicina social latino-americana a noção de problemas ambientais e de problemas de saúde éampliada. A pesar dos avanços, a análise de dados sobre grupos de pesquisa, a produção de teses e dissertações e a publicação de artigos científicos revelam que o campo da saúde coletiva ocupa um papel marginal na pesquisa sobre o tema problemas ambientaise a pesquisa e a produção das ciências sociais respondem por uma parcela muito pequena. 0 quadro atual impõe a necessidade de se avançar quantitativa e qualitativamente na pesquisa e produção científica da saúde coletiva sendo urgente no que se refere às ciências sociais e, particularmente, nas ciências sociais em saúde.

Palavras-chave Problemas ambientais, Ciências sociais, Saúde coletiva 


\section{Introdução}

De acordo com Andler (1987), a caracterização de uma atividade fundada sobre a noção de problema não pode ser completa sem a presença do conceito de solução. Esta noção certamente se aplica aos problemas ambientais, reais ou potenciais, que afetam à todos no planeta. Os problemas ambientais são problemas eminentemente sociais, gerados e atravessados por um conjunto de processos sociais (Leff, 2000) e, como tais, só vieram à tona porque, como ambientes criados, não se encontram alheio à vida social humana, mas são completamente penetrados e reordenados por ela, confundindo atualmente o que é "natural" com o que é "social" (Giddens, 1990; Beck, 1997).

Como observa Samaja (2000), o termo "problema" só tem campo de aplicação nos sistemas vivos e nos processos humanos, pois são os que enfrentam problemas em sua existência e realizam escolhas que lhes permitem mudar de uma situação para outra. Por essa razão, a noção de "problemas de saúde" compõe uma ordem descritiva que serve para qualificar estados possíveis nos indivíduos vivos em toda a extensão da biosfera. A pesar disso, no que diz respeito aos problemas ambientais, que são simultaneamente problemas de saúde, pois afetam os seres humanos e as sociedades em múltiplas e simultâneas escalas e dimensões, o que se assiste é um movimento atual deformalização dos problemas que, na maioria das vezes, reduz os mesmos ao conceito de resolução através do cálculo e do tratamento da informação na lógica das ciências naturais e engenharias.

Retomando Andler (1987), é importante sublinhar que a escol ha de um problema é, irredutivelmente, uma escolha. Então, se consideramos que não existe um único ambiente, o ambiente construído e descrito pelas ciências naturais e engenharias, mas sim uma variedade de ambientes constituídos histórica, geográfica, social e culturalmente, surge então a necessidade de se considerar que um problema ambiental corresponde à uma multiplicidade de problemas ambientais simultâneos, que envolvem diferentes e conflituosas noções de sociedade. Problemas que necessariamente envolvem processos sociais, políticos, econômicos e culturais, bem como uma multiplicidade de atores sociais com diferentes noções e inter esses acerca dos mesmos e das formas de resolução que poderão ser encaminhadas. I sso implica que resolução do(s) problema(s) somente através do cálculo e do tratamento da informação na lógica das ciências naturais e engenharias será sempre limita$\mathrm{da}$, necessitando-se de uma presença maior das ciências sociais na compreensão e busca de solução para o(s) mesmo(s).

Se consideramos a especulação de Schackley et al. (1996), de que as considerações dominantes de um realismo ambiental por parte das ciências naturais e engenharias possuem poderosos efeitos sobre nossas construções sociais acerca do que é problema e do que é o ambiente, podemos considerar que o caminho inverso é igualmente verdadeiro. Como observa Giddens (1990), em condições de modernidade, 0 mundo social nunca pode formar um ambiente estável em termos de entrada de conhecimento novo sobre seu caráter e funcionamento. Para o autor, o conhecimento novo (conceitos, teorias, descobertas) não torna simplesmente o mundo social mais transparente, mas altera sua natureza, projetando-a para novas direções, afetando tanto a natureza socializada, bem como as próprias instituições sociais.

A noção de problemas ambientais não só permite uma maior incorporação das ciências sociais para a sua compreensão e resolução, mas se encontra mais em consonância com o projeto da saúde coletiva. Essa noção permite considerar que no projeto da saúde coletiva não só a saúde surge como uma conquista social e um direito universal associados à qualidade e à proteção da vida, como afirma M inayo (1997), mas também o ambiente. $N$ esta perspectiva, 0 desenvolvimento da ciência e da tecnologia para a compreensão dos problemas ambientais, que são simultaneamente problemas de saúde, deverá, como considera M inayo (1997), estar ao serviço do sentido social, político e de direito universal, o que inclui a eqüidade.

\section{0 meio ambiente na saúde pública}

A preocupação com os efeitos na saúde provocados pelas condições ambientais é evidente desde a Antigüidade, envolven do problemas tais como os efeitos do clima no balanço dos humores do corpo, os miasmas, as sujeiras e os odores. Assim, sempre esteve presente nos diferentes discursos e práticas sanitárias que se constituíram como respostas sociais às necessidades e aos problemas de saúde. Essa preocupação parece se acentuar particularmente entre meados do século 18 e meados do século 19, quando os problemas ambientais sobre a saúde estive- 
ram associados aos efeitos do rápido e intenso processo de industrialização e urbanização que passaram a incidir nas condições de vida e trabalho. Nesse período, as preocupações e estraté gias sanitárias tinham por base a teoria dos miasmas, para a qual as sujeiras externas e os odores detectáveis deveriam ser reduzidos ou eliminados para deter a disseminação das doenças. A higiene é introduzida como uma estratégia de saúde para as populações, envolvendo a vigilância e o controle dos espaços urbanos (ruas, habitações, locais de lixos, sujeiras e toxicidade) e grupos populacionais (pobres, minorias étnicas e as classes trabal hadoras) considerados sujos e perigosos. 0 ambiente das cidades era identificado como "objeto medicalizável", havendo a tendência de se patologizar determinadas regiões e lugares, que, habitados pelos pobres, minorias étnicas e classes trabal hadoras, deveriam ser evitados pelos "cidadãos decentes" (burguesia) (Petersen \& Lupton, 1996).

Entre meados e o final do século 19 são bastante intensos os impactos da Revolução Industrial sobre as condições de vida e saúde das populações. Principalmente nos países europeus, onde houve maior desenvolvimento nas relações industriais de produção (Inglaterra, França e Alemanha), ocorreu também uma maior organização das classes trabal hadoras, com 0 aumento da sua participação política. Os temas relativos à saúde são incorporados na pauta das reivindicações dos movimentos sociais e surgem propostas de compreensão da crise sanitária como fundamentalmente um processo político e social, recebendo a denominação de medicina social. Ao contrário do higienismo para o qual 0 ambiente era um objeto medicalizável através de um conjunto de normatizações e preceitos a serem seguidos e aplicados no âmbito individual, a participação política é concebida como principal estratégia de transformação da realidade de saúde (Paim \& Almeida FiIho, 1998). Desbaratado no plano político o movimento da medicina social, estrutura-se, princi pal mente na Inglaterra e nos Estados U nidos, o movimento do sanitarismo como uma resposta estreitamente integrada à ação do Estado no âmbito da saúde. Como observam Paim \& Almeida Filho (1998), o discurso ea prática dos sanitaristas sobre os problemas de saúde eram fundamentalmente baseados na aplicação de tecnologia e em princípios de organização racional para a expansão das atividades profiláticas, destinadas principalmente aos pobres e setores excluídos da população. No que se refere aos problemas ambientais, o saneamento e 0 controle de vetores constituíram a principal estratégia desse movimento, direcionada para 0 controle de doenças rel acionadas às precárias condições sanitárias ( Gochefeld \& Goldstein, 1999). 0 advento do paradigma microbiano nas ciências básicas da saúde representou um grande reforço a este movimento que, tornado hegemônico e batizado de saúde pública, reorienta as diretrizes dos discursos e das práticas ocidentais no campo da saúde social (Paim \& Almeida Filho, 1998). Com o paradigma microbiano, 0 ambiente de foco dos discursos e das práticas da saúde pública é o doméstico, que deveria ser purificado, limpo e areado, sendo isto considerado vital para a saúde dos seus habitantes, particularmente as crianças (Petersen \& Lupton, 1996).

A ampliação da compreensão dos problemas ambientais como não somente restritos aos aspectos de saneamento e controle de vetores, bem como a recuperação da dimensão política e social dos mesmos pode, em grande parte, ser atribuída às questões que passaram a ser colocadas pelo movimento ambientalista, que, definido como tal, tem sua existência identificada desde os anos 50, passando a ganhar força somente nos anos 60 e 70. As ameaças e os perigos ambientais para a saúde pública, provocadas principalmente pela poluição química e radioativa, são compreendidas como de maior escala, tendo se multiplicado e estendido no espaço - indo além dos ambientes locais da casa, da vila ou da cidade - e no tempo - com o alcance dos efeitos futuros sobre a saúde e a vida no planeta ( $\mathrm{Pe}$ tersen \& Lupton, 1996).

A partir do último quartel do século 20 , a preocupação com os problemas ambientais tornou-se proeminente em muitos países e resultou em duas grandes conferências mundiais sobre o tema, organizadas pela O rganização das Nações U nidas (ONU), a deEstocolmo em 1972 ea do Rio em 1992. Em paralelo, emerge uma Nova Saúde Pública (NSP) que tem como estratégia mudar o foco das práticas centradas principalmente nos aspectos biomédicos da atenção para uma compreensão preventiva do estado de saúde, passando a direcionar muita de sua atenção para as dimensões ambientais da saúde (Petersen \& Lupton, 1996). Emblemáticos deste processo são: Relatório Lalonde em 1974, que define as bases para o movimento de Promoção da Saúde e em que são incorporadas questões como a criação de ambientes favoráveis à saúde; o Projeto Cidades Saudáveis lançado em 
1986 pela Organização M undial da Saúde; a definição na Agenda 21 da saúde ambiental como prioridade social para a promoção da saúde.

\section{Os problemas ambientais na saúde coletiva e sua incorporação pelas ciências sociais}

De acordo com M inayo et al. (1999), no Brasil, a preocupação com os problemas ambientais, as características socioeconômicas do desenvolvimento e a interface de ambos com a saúde coletiva pode ser situada desde o início do século, através do trabalho pioneiro de $\mathrm{O}$ swaldo $\mathrm{Cruz}$ e dos sanitaristas que o seguiram. Embora mais voltados para a problemática na Fundação Oswaldo Cruz, os autores identificam três paradigmas básicos presentes nos estudos sobre a interface entre problemas ambientais e saúde, sendo estes: o biomédico, com origens na parasitologia clássica; o da relação sanemanto-ambiente, com origens no saneamento clássico; 0 da medicina social, que tem suas origens nos anos 70 e é a referência para a saúde coletiva.

Para Tambellini \& Câmara (1998), do ponto de vista institucional, as preocupações com os problemas ambientais tradicional mente relacionadas à saúde foram, ao longo do século 20, uma preocupação quase que exclusiva das instituições voltadas ao saneamento básico (água, esgoto, lixo, etc.). Para os autores, somente na década de 1970, com o agravamento dos problemas ambientais causados pelo crescimento industrial, ocorre uma ampliação das instituições, com a criação, por exemplo, de órgãos ambientais nos estados do Rio de J aneiro (Feema) eSão Paulo (Cetesb), mas sem vínculo direto com o sistema de saúde. É importante lembrar que em 1972 era realizada a Conferência de Estocolmo, primeira grande reunião mundial sobre a relação entre ambiente e desenvolvimento.

Freitas et al. (1999) e Porto (1998), procurando contextualizar a interface entre a questão ambiental e a saúde no país, consideram que somente a partir da década 1980 é que começaram a surgir condições jurídicas e institucionais para ações de controle do meio ambiente mais consistentes e efetivas. Como exemplo citam a lei 6.938, de 1981, que estabeleceu a Política Nacional de M eio Ambiente e criou o Sistema $\mathrm{Na}$ cional de M eio Ambiente e o Conselho Nacional de $\mathrm{M}$ eio Ambiente. $\mathrm{Na}$ Constituição Federal promulgada em 1988 novos avanços ocorreram, enunciando-se no artigo 228 do capítulo
VI (Do M eio Ambiente) que todos têm direito ao meio ambiente ecologicamente equilibrado, bem de uso comum do povo e essencial à sadia qualidade de vida, impondo-se ao Poder Público o dever de defendêlo e à coletividade de preservá-lo para as presentes e futuras gerações.

Nesse período, entre os anos 70 e 80, acontece também o desenvolvimento do movimento da saúde coletiva, que situava-se no âmbito dos movimentos pela democratização das formações sociais latino-americanas. Partindo da compreensão que a saúde da população resulta da forma como se organiza a sociedade, em suas dimensões política, econômica e cultural, esse movimento propunha mudanças em direção tanto à democratização da sociedade, como das práticas de saúde, implicando isso a sua própria reorganização (Paim \& Almeida Filho, 1998; Paim, 2001).

Embora os anos 70 e 80 tenham sido importantes na incorporação da temática ambiental, somente nos anos 90, com a Conferência do Rio em 1992 e a publicação da Agenda 21, com um capítulo dedicado à saúde, é que começou a se assistir uma incorporação mais ampla e efetiva da temática ambiental na saúde coletiva (Freitas et al., 1999; Porto, 1998). M arco desse processo na saúde coletiva foi a organização, pela Escola N acional de Saúde Pública, dos dois volumes sobre saúde, ambiente e desenvolvimento (Leal et al., 1992a e 1992b). N esse mesmo ano, a OPAS decidiu organizar, em outubro de 1995, uma conferência pan-americana sobre saúde, ambiente e desenvolvimento. Em 1994 iniciaram-se as ações do governo brasileiro de preparação para esta conferência e em 1995 foram realizadas quatro oficinas de trabal ho (Brasília, Recife, Rio de Janeiro e Belém), envolvendo membros de um grupo de trabalho de diversos ministérios e OPAS, coordenado pelo M inistério da Saúde. Das oficinas participaram demais órgãos públicos afins com a temática, instituições acadêmicas, entidades da sociedade civil e organizações não-governamentais. No final dos anos 90 , através do projeto Vigisus, inicia-se a estruturação e a institucionalização da vigi lância ambiental no âmbito do M inistério da Saúde, sendo publicado em maio de 2000 o decreto 3.450 que estabeleceu no Cenepi a gestão do sistema nacional de vigilância ambiental.

Embora ainda em fase de andamento, resultados preliminares de um projeto de pesquisa que 0 autor vem realizando, denominado "A pesquisa científica em saúde ambiental no Brasil - 1992-2001", contribuem para compreender 
este quadro do ponto de vista da pesquisa, particularmente no campo da saúde coletiva.

No levantamento dos grupos de pesquisa (Diretório dos Grupos de Pesquisa, versão 4.0) que tem como pal avra-chave da linha de pesquisa a questão "ambiental", encontrou-se um total de 824 grupos, sendo que destes, apenas 32 (3.9\%) foram identificados como pertencentes a área predominante (AP) de saúde coletiva. Restringindo o levantamento incluindo a palavra-chave saúde, obteve-se o seguinte resultado: a) para "saúde" e "ambiental", encontrou-se um total de 69 grupos, sendo 25 (36\%) identificados como pertencentes a AP de saúde coletiva; b) para "saúde" e "ambiente", encontrou-se 78 grupos, sendo 27 (34\%) identificados como pertencentes a AP de saúde coletiva.

Todos os grupos de pesquisa identificados nos três levantamentos como pertencentes à AP de saúde coletiva e suas respectivas instituições encontram-se listados no quadro 1. D os 42 grupos listados, pode-se identificar apenas 5 (todos em negrito e correspondendo à $12 \%$ do total) como próximos ou diretamente identificados com temas de interesse para as ciências sociais: 1 no campo da filosofia (bioética e ética); 2 no campo da educação; 1 no campo da antropologia (impactos da difusão de tecnologias na qualidade de vida e cultura); 2 específicos sobre as ciências sociais.

N os dois levantamentos em que se cruzou a palavra-chave "saúde" com "ambiental" e "ambiente", identificamos 6 grupos que tiveram AP diretamente relacionada às disciplinas de interesse para as ciências sociais em saúde listadas por Canesqui (1998), cujo resultado é apresentado com o nome do grupo e a instituição entre parênteses. $\mathrm{Na}$ sociologia, o Centro de Pesquisas e Estudos Agrários (U nesp). $\mathrm{Na}$ antropologia, o Dinâmica Sociocultural, Gestão Ambiental e Desenvolvimento: U ma Antropologia da A ção entre os Ticuna do Alto Solimões (UFRJ). $\mathrm{N}$ a demografia, Demografia e Políticas Públicas (Unicamp). Na ciência política, o Grupo de Estudos e Pesquisas Eneida de M orais Sobre M uIher e Relações de Gênero (UFPA). Embora o serviço social não faça parte da lista de Canesqi (1998), foram identificados 2 como de interesse para as ciências sociais em saúde, sendo: Núcleo de Pesquisa e Estudo - Estado Sociedade e Cidadania (UCG) e o Programa de Estudos do Trabalho e da Reprodução Social (UERJ).

No levantamento de teses e dissertações produzidas no Brasil entre os anos 1980 e 2000, identificadas com a palavra chave "ambiente", real izado na base de dados Literatura LatinoAmericana e do Caribe em Ciências da Saúde (LILACS), encontrou-se um total de 305 títulos. Para melhor vislumbrar o crescimento da produção acadêmica sobre o tema, agrupou-se as teses e dissertações por triênio, conforme pode se verificar na figura 1.

Conforme pode se verificar, houve um nítido crescimento da produção sobre os problemas ambientais, particularmente a partir da década de 1990, principalmente da segunda metade em diante, período que concentrou mais da metade da produção (54\%) de todo o período.

$\mathrm{N}$ o que se refere especificamente às ciências sociais, procurou-se pelo menos identificar as teses e dissertações mais diretamente relacionadas às mesmas através do título e da abordagem metodológica adotada e descrita no resumo. Dos 305 títulos, foram encontrados 17 (5.6\%), distribuídos pelos seguintes temas: educação (3); políticas públicas (4); percepções e representações sociais (3); aspectos históricos (2); desi gualdades sociais (1); geografia crítica (1); filosofia (1); sociologia da ciência (1); participação popular (1). 0 maior volume de teses ou dissertações concluídas foi no ano de 1996, com 5; em seguida vem 2000, com 3; e, 1990, 1992 e 1998, com 2. Para os outros anos (1983, 1985, 1993 e 1999) foram identificadas apenas uma para cada ano.

Em termos percentuais pode-se considerar muito baixo os $5.6 \%$ de teses e dissertações identificadas com as ciências sociais, devendose observar que as relacionadas à percepção e representações sociais representaram quase $1 / 4$ do pequeno universo de 17 títulos.

No levantamento realizado nas três principais revistas científicas no campo da saúde coletiva (Ciência e Saúde Coletiva, Cadernos de Saúde Pública e Revista de Saúde Pública) no período 1992-2001, o critério adotado foi selecionar todos os artigos que, através do título ou palavra-chave, se identificaram como referentes ao tema "ambiente" ou "ambiental". Foram encontrados 94 artigos, distribuídos de modo bastante irregular por ano (Figura 2). Conforme pode se verificar na figura 2 , houve uma concentração maior de artigos publicados no ano de 1998, estando o fato associado à publicação de um número especial da revista Ciência e Saúde Coletiva (volume 3 , número 2), dedicado ao tema "saúde e ambiente no processo de desenvolvimento".

Do total de artigos, identificamos apenas 9 $(9,6 \%)$ sobre temas e abordagens de interesse 
Quadro 1

Grupos de pesquisa no campo da saúde coletiva, identificados por título e instituição

\begin{tabular}{|c|c|}
\hline Nome do grupo & Instituição \\
\hline Avaliação Integrada e Participativa de Riscos Tecnológicos Ambientais & Fiocruz \\
\hline Avaliação e Gestão de Riscos Ambientais & CNEN \\
\hline Bioética e Ética A plicada à Saúde & Fiocruz \\
\hline Centro de Controle de Intoxicações de M aringá & UEM \\
\hline Centro de Documentação, Pesquisa e Formação em Saúde e Trabalho & UFRGS \\
\hline Doenças Emergentes e Ambientais na Região Centro-O este & UFMS \\
\hline Educação para a Saúde & Fiocruz \\
\hline Epidemiologia & Unisinos \\
\hline Epidemiologia do Câncer Ambiental & USP \\
\hline Estudo dos Fatores Físicos do M eio A mbiente Sobre a Saúde dos I dosos & PUC-RS \\
\hline Grupo de Epidemiologia de Londrina & UEL \\
\hline Grupo de Estudos em Epidemiologia & IS \\
\hline Grupo de Estudos da Problemática dos Resíduos de Serviços de Saúde & USP \\
\hline Grupo de Estudos de Gerência e Ensino em Saúde & UFF \\
\hline $\begin{array}{l}\text { Grupo de Pesquisa em Ciências Sociais, Extensão Rural, Saúde } \\
\text { eAmbiente- Diagnóstico e Avaliação de Projetos Sociais }\end{array}$ & USP \\
\hline Impacto das Condições do Ambiente Sobrea Saúde & UFMG \\
\hline Informação em Saúde & Fiocruz \\
\hline $\begin{array}{l}\text { Investigação de M ercúrio na Região Amazônica em Populações H umanas } \\
\text { ena Biota Aquática deÁreas Sujeitas à Poluição Ambiental }\end{array}$ & IEC \\
\hline Investigação sobre a N ormalidade da Região Amazônica quanto & IEC \\
\hline à Presença de M ercúrio em Populações Humanas e na Biota Aquática & \\
\hline Laboratório de Educação em Ambiente e Saúde & Fiocruz \\
\hline M alária na Amazônia & INPA \\
\hline M eio Ambiente eSaúde & CEDEC \\
\hline M eio Ambiente/Vigilância Epidemiológica & $\mathrm{CIP}$ \\
\hline M odo de Vida, Qualidade de Vida e Saúde & UFBA \\
\hline M onitoramento Ambiental & UNIPAR \\
\hline Núcleo de Apoio à População Ribeirinha da Amazônia & USF \\
\hline Núcleo de Epidemiologia & UEFS \\
\hline Núcleo de Estudos em Ciências Sociais, Ambiente e Saúde & UFBA \\
\hline Núcleo Interdisciplinar de M eio Ambiente & UFBA \\
\hline Núcleo de Pesquisas e Estudos em Saúde Coletiva & UFMG \\
\hline Programa de M udanças Ambientais G lobais e Saúde & Fiocruz \\
\hline Repercussões da Difusão de Tecnologias na Qualidade de Vida e Cultura & Fiocruz \\
\hline SaúdeAmbiental & Fiocruz \\
\hline Saúde Ambiental em Área U rbana & IEC \\
\hline Saúde Coletiva & UEPB \\
\hline Saúde e Ambiente & $\mathrm{CIP}$ \\
\hline Saúde e Ambiente & UNICSUL \\
\hline Saúde e M eio A mbiente & UNIFESP \\
\hline Saúde eT rabalho & CIP \\
\hline Saúde, Trabalho e M eio Ambiente & UFPB \\
\hline Saúde, Trabalho e M eio Ambiente & UFPA \\
\hline Substâncias Q uímicas: Impacto Sobre a Saúde e o Ambiente & Fiocruz \\
\hline Trabalho, Ambiente eSaúde & UFRJ \\
\hline
\end{tabular}

Fonte: CNPq - Diretório dos Grupos de Pesquisa, versão 4.0 
Figura 1

T eses e dissertações sobre a questão “ambiental” publicadas entre 1980 e 2000

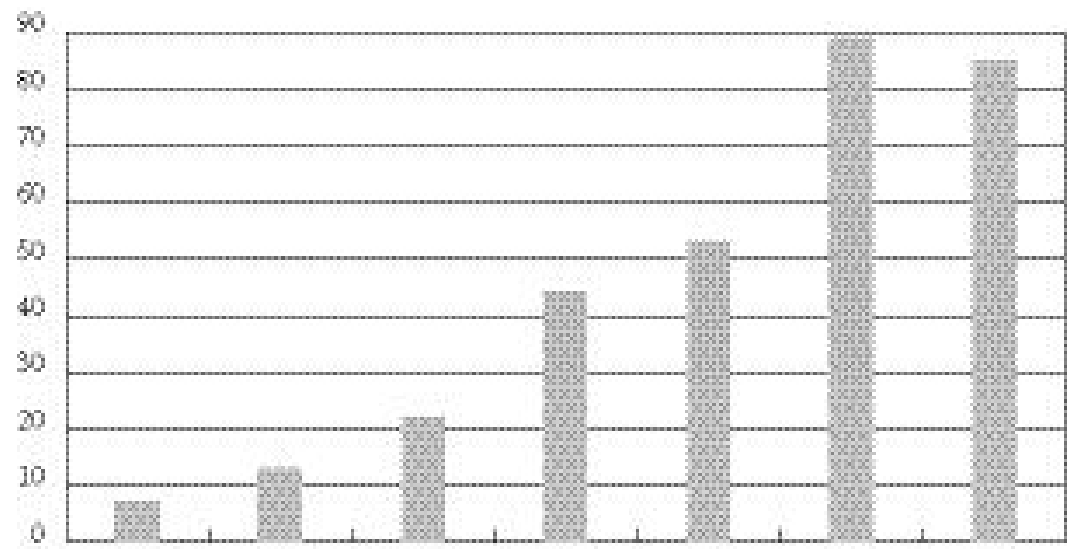

1980-1982 1963-1905 1986-1988 1989.1991 1992-1994 1995.1997 1998.2000

Fonte: Literatura Latino-Americana e do Caribe em Ciências da Saúde - Biblioteca Virtual em Saúde

\section{Figura 2}

Artigos publicados em revistas científicas brasileiras, entre 1992 e 2001, identificados com o tema "ambiente" ou "ambiental"

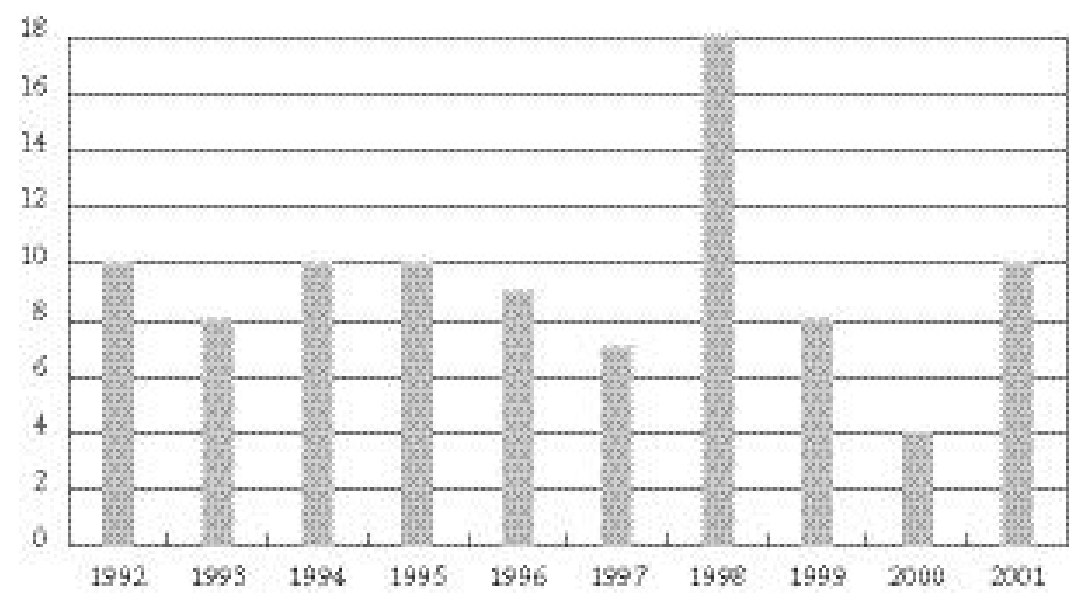

Fonte: Cadernos de Saúde Pública, Revista de Saúde Pública, Ciência e Saúde Coletiva 
para as ciências sociais, distribuídos do seguinte modo: educação (3); ineqüidades sociais e a saúde dos trabalhadores (1); a economia ambiental e as tomadas de decisões (1); os movimentos sociais e as questões éticas (1); os riscos industriais e tecnológicos ambientais (2); 0 saber ambiental (1); as políticas públicas (1). Destes, 3 (Schall, 1994; Porto \& Freitas, 1997; Duval, 1998), tendo como base as ciências sociais, apontam para a crítica das ciências biomédicas e engenharias, propondo a construção de um conhecimento participativo e inter ou transdisciplinar. É interessante observar que as publicações que tiveram como tema a educação corresponderam à aproximadamente $1 / 3$ do total da produção identificada com as ciências sociais.

Para encerrar a análise de dados referentes à pesquisa e produção referente aos problemas ambientais e saúde discutiremos os dados apresentados no artigo de M inayo et al. (1999). Para o ano de 1997 os autores cadastraram 151 projetos de pesquisa sobre saúde e ambiente em andamento na Fiocruz. A partir de uma lista com as áreas de conhecimento mais citadas no cadastro, os autores identificaram 32 áreas citadas no mínimo cinco vezes (a epidemiologia foi a que recebeu maior número de citações, com 45 vezes), resultando em um total de 434 citações. Das áreas do conhecimento identificadas, selecionamos aquel as mais diretamente relacionadas às ciências sociais, resultando em: educação (22), antropologia (16), sociologia (13), geografia (9) e história (6). M esmo considerando que a maioria dos projetos se apresenta como interdisciplinar, não pode deixar de se notar o baixo percentual de citações de áreas relacionadas às ciências sociais ( $15 \%)$, sendo que deste universo de 66 citações, $1 / 3$ encontravase relacionado à educação.

Das informações apresentadas nos parágrafos anteriores são indicadores de que a institucionalização da temática ambiental de modo amplo e efetivo na saúde coletiva só ocorreu nos anos 90, sendo o projeto Vigisus e a figura 1 relacionados à produção de teses e dissertações, indicativos disto. Constatamos que embora os problemas ambientais quase sempre sejam, simultaneamente, problemas de saúde, a esmagadora maioria dos grupos de pesquisa que tem como tema de investigação a questão "ambiental" (96,1\%) se situa fora da AP da saúde coletiva. M esmo quando restringimos a pesquisa sobre os grupos de pesquisa, incluindo a palavra-chave "saúde", constatamos que os grupos que têm como AP a saúde coletiva corres- pondem a aproximadamente $1 / 3$ do total, estando os outros $2 / 3$ distribuídos em uma diversidade de grupos, em uma multiplicidade de APs (por exemplo: engenharia sanitária, ecologia, geociências, química, enfermagem, medicina, educação e demografia).

$\mathrm{N}$ o que se refere especificamente à participação das ciências sociais, podemos considerar o percentual de grupos diretamente identificados com as mesmas ainda muito baixo na AP da saúde coletiva. Fora da AP da saúde coletiva, mas identificados com as inter-relações entre as questões "ambiente" ou "ambiental" com a "saúde", surgem seis grupos de APs relacionados às ciências sociais. Do ponto de vista do potencial de intercâmbio acadêmico e da constituição de redes de pesquisa social em saúde e ambiente, esses grupos são de grande importância no sentido de ampliar e fortalecer a pesquisa nas ciências sociais na saúde coletiva. Até mesmo porque o quadro que se apresenta sobre a incorporação dos problemas ambientais nas ciências sociais na área da saúde coletiva é de uma capacidade maior de se organizar em grupos de pesquisa ( $12 \%$ ) ou projetos de pesquisas ( $15 \%$ no levantamento feito para a Fiocruz), do que de produção de artigos científicos ( $9,6 \%$ nas 3 revistas) ou teses edissertações (5.6\% no LILACS).

A pesar de desde os anos 70 assistirmos avanços no ambiental ismo nos países industrializados e o desenvolvimento da saúde coletiva no Brasil, ambos os movimentos trazendo novamente à tona as dimensões sociais, políticas, econômicas, culturais e éticas dos problemas ambientais e sanitários, o que se verifica, na prática, éuma predominância das ciências naturais e engenharias na organização da pesquisa e produção do conhecimento sobre o tema. Embora as dimensões sociais, políticas, econômicas, culturais e éticas não sejam exclusividade das ciências sociais, é preocupante que mesmo no campo da saúde coletiva verifique-se que há poucos grupos de pesquisa organizados e que a produção científica ainda seja bastante pequena.

\section{Os problemas ambientais nas ciências sociais}

Conforme demonstrado no item anterior, a organização de grupos de pesquisa e a produção científica sobre problemas ambientais nas ciências sociais no campo da saúde coletiva é ainda pequena. Essa característica não se restringe às ciências sociais em saúde, mas reflete uma ten- 
dência geral. Os resultados preliminares da pesquisa que vem sendo conduzida pelo autor, citada no item anterior, permitem vislumbrar melhor este quadro no Brasil.

Para os 824 grupos de pesquisas que têm como tema de pesquisa a questão "ambiental", adotamos dois enfoques, um restrito, baseado no estudo de Vieira (1995) sobre o mapeamento e avaliação da pesquisa em ciências sociais no Brasil (período 1980-1990), e outro amplo, mais próximo do nosso interesse e tendo como referência o estudo de Canesqui (1998) sobre três décadas de ensino e pesquisa das ciências sociais em saúde no Brasil. No enfoque restrito, dentre os 824 grupos, os resultados foram: geografia (22), antropologia (8), economia (16), sociologia (9), ciência política (1). Totalizaram 56 grupos e corresponderam a apenas $6.8 \%$ do total. No enfoque amplo, os resultados foram: educação (32), história (3), filosofia (2), demografia (1). Totalizaram 38 grupos. Somados os dois grupos, ampliamos para 94 , alcançando com as 9 disciplinas incluídas apenas $11.4 \%$ dos 824 grupos. Só para se ter uma idéia mais clara dos desequilíbrios entre as diferentes disciplinas envolvidas com a temática ambiental, a soma das cinco que mais possuem grupos, a geociências (100) equímica (79) nas ciências exatas e da terra, a agronomia (63) nas ciências agrárias, a ecologia (61) nas ciências biológicas, ea engenharia sanitária (42) nas engenharias, totalizaram 345 grupos, correspondendo à $42 \%$ do total.

Para Leff (2000), tendo por base um diagnóstico sobre os programas de formação ambiental em nível universitário na América Latina e Caribe e um estudo sobre a incorporação da dimensão ambiental nas ciências sociais, estas ciências se encontram entre as disciplinas mais resistentes a transformar seus paradigmas de conhecimento e a abrir seus temas privilegiados de estudo com relação à problemática ambiental.

M acnaghten \& U rry (1998) consideram que negligenciamento do social na literatura ambiental é parcialmente atribuível a própria trajetória do desenvolvimento das ciências sociais, sendo mais acentuado na sociologia. Para os autores, baseia-se em uma forte e indesejável divisão entre o mundo dos fatos sociais e o mundo dos fatos naturais, entre sociedade e nature za, contribuindo para que as ciências sociais se preocupassem menos com as formas biológicas ou ambientais. No que concerne especificamente à sociologia, Leff (2000) observa que esta se desenvolveu dentro de enfoques e problemas teóricos que têm tido dificuldade de internalizar facilmente os processos socioambientais emergentes, tanto por sua complexidade, como por seu caráter de novidade e pelas inter-relações entre processos de ordem física, biológica esocial.

Além dos aspectos anteriormente apontados, M acnaghten \& U rry (1998) chamam a atenção para a concepção hegemônica de um realismo ambiental. N esta concepção, 0 ambienteé uma entidade real em si, passível de ser pesquisado por uma ciência capaz de fornecer uma compreensão reificada do mesmo, produzindo resultados observáveis e não ambíguos. Isto possibilita não só mensurações, mas também a possibilidade de se avaliar todas as medidas necessárias para se corrigir os danos tendo por base a mesma ciência que os gerou. Para esta concepção, uma vez que a realidade derivada da pesquisa científica transcende os padrões transitórios e superficiais da vida cotidiana, a incorporação da análise dos processos sociais e práticas institucionais, bem como da experiência humana, ocupa um papel menor (Shackley et al., 1996; M acnaghten \& U rry, 1998; Leff, 2000).

De um modo geral, na concepção hegemônica do realismo ambiental, quando as ciências sociais são chamadas para a pesquisa e o ensino sobre os problemas ambientais, seu papel fica restrito a identificar as causas sociais, os impactos sociais e as respostas sociais aos problemas ambientais inicialmente descritos de forma acurada pelas engenharias e ciências naturais. Diversos cientistas sociais (Shackley et al., 1996; M acnaghten \& U rry, 1998; Leff, 2000) têm chamado a atenção para essa questão através do exemplo de um problema ambiental global, como os programas internacionais de pesquisa sobre mudanças ambientais globais. Esses programas tendem a minimizar ou reduzir a especificidade dos processos sociais em suas análises e a atribuir um papel secundário às ciências sociais em suas análises, que deverá, no máximo, deixando à margem a pesquisa sobre os conflitos sociais e as inúmeras estratégias políticas dos diferentes atores envolvidos, formular respostas sociais adequadas aos problemas enunciados a partir de um grande conjunto de evidências oriundas das ciências naturais e engenharias.

Vieira (1995), em seu mapeamento da produção das ciências sociais sobre a problemática ambiental no Brasil, revela ser dominante a concentração do esforço de pesquisa acerca do tema "avaliação dos impactos socioambientais 
da dinâmica de desenvolvimento socioeconômico". Foi o único tema de pesquisa comum a maioria das disciplinas que identificou (antropologia, sociologia, ciência política, geografia humana), sendo o tema "avaliação de políticas de desenvolvimento e gestão ambiental" encontrado somente na ciência política. Outros temas de pesquisa que identificou com as disciplinas selecionadas foram: análise do movimento ecologista (sociologia); educação, participação e meio ambiente (sociologia e ciência política); ecologia e relações internacionais (ciência política); análise espacial e planejamento (geografia humana).

$O$ autor (Vieira, 1995) chama a atenção para duas lacunas importantes na produção das ciências sociais, as quais devem ser superadas para permitir seu avanço na compreensão e solução dos problemas ambientais. A primeira se refere à reflexão epistemológica estar aquém das necessidades de operacional ização efetiva de enfoques interdisciplinares que permitam às ciências sociais avançar tanto no plano do conhecimento teórico, quanto no da intervenção social e política. Só a sociologia e as ciências políticas foram identificadas pelo autor tendo como tema a pesquisa sobre as repercussões da problemática ambiental no seu campo teórico e metodológico. A segunda se refere à ênfase nos estudos sobre avaliação dos impactos destrutivos, contrastando com a escassez de estudos acerca da viabilidade de estratégias alternativas de desenvolvimento socialmente justas, economicamente viáveis, ecologicamente prudentes e politicamente emancipadoras.

No que se refere à segunda lacuna apontada no parágrafo anterior, deve-se considerar que a viabilidade de estratégias alternativas, transformadas em projetos de intervenção, envolve, como argumenta Vieira (1995), elevado número de fatores, de natureza não-linear nas suas inter-relações e que são de difícil captação. Para o autor, a gestão da complexidade é, então, o principal obstáculo a ser enfrentado pelos analistas e planejadores. Com a nova ênfase sobre a complexidade dos problemas ambientais tem ocorrido uma tentativa cada vez maior de introduzir "ferramentas" das ciências sociais nos processos decisórios, consideradas menos reducionistas ( processo causa-efeito linear, unidimensional e determinístico) do que os métodos de análise custo-benefício comumente empregados (Schackley et al., 1996).

Porém, como argumentam Schackley et al. (1996), a transformação das ciências sociais em "ferramenta" na análise, planejamento e gestão dos problemas ambientais pode limitar seu próprio emprego, significando um filtro em favor das abordagens que privilegiem argumentos considerados mais realistas para as ciências naturais e engenharias, bem como para os tomadores de decisões. N essas situações, os cientistas sociais têm o seu papel reduzido ao de engenheiros sociais, capazes de manipular e fixar a sociedade de modo a facilitar a implementação de desenvolvimento sustentável definido essencialmente em termos técnicos a partir das ciências naturais e engenharias ( $M$ acnaghten \& Urry, 1998).

O desafio que se coloca para as ciências sociais é que a tendência crescente de incorporação de suas teorias e metodologias como "ferramenta" para a compreensão e solução dos problemas ambientais pode apenas parcialmente significar a superação das lacunas que Vieira (1995) apontou e limitar sua incorporação de modo amplo, crítico e reflexivo. Abordagens mais contextualizadas e relativistas, bem como críticas dos padrões de trabalho, produção e consumo que contribuem para a degradação socioambiental tenderiam a ser marginalmente incorporadas por duas razões. Em primeiro lugar, porque a idéia de que não há uma única noção de meio ambiente, mas somente ambientes histórica, geográfica e socialmente constituídos coloca em xeque a concepção hegemônica do realismo ambiental tanto nas ciências naturais e engenharias, como nas diferentes instituições públicas e privadas em que têm se dado as decisões que afetam nossas vidas. Em segundo lugar, abordagens críticas que apontem para a necessidade de uma transformação ecológica das sociedades industriais tenderiam a ser consideradas desestabilizadoras das estruturas sociais, políticas e econômicas dominantes, desafiando inclusive o papel do Estado na regulação ambiental das atividades de trabalho, produção e consumo (Petersen \& Lupton, 1996; Schackley et al., 1996; M acnaghten \& U rry, 1998).

\section{Conclusão}

0 ambiente sempre esteve presente nos discursos e práticas sanitárias. M as, foi somente com a intensificação do processo de industrialização e urbanização, 0 aumento da participação política da classe trabal hadora e a incorporação dos temas relacionados à saúde na pauta de reivindicações dos movimentos sociais, que os pro- 
blemas ambientais passaram a ser compreendidos como resultantes de processos fundamentalmente políticos e sociais. É nesse contexto de fortalecimento dos movimentos sociais que emerge a medicina social no século 19 , para a qual a participação política era concebida como principal estratégia de transformação da realidade de saúde. No século 20 , a recuperação da dimensão social e política dos problemas ambientais ocorre a partir do crescimento dos movimentos contestatórios e ambientalistas entre os anos 60 e 70.

Petersen \& Lupton (1996) observam que no último quartel do século 20 chegou mesmo a existir uma proximidade entrea NSP (da qual derivam movimentos importantes hoje no que se refere à problemática ambiental no campo da saúde, como o movimento de promoção da saúde e o projeto cidades saudáveis) e o movimento ambientalista. A proximidade de ambos era derivada do fato de considerarem tanto o adoecimento do planeta por conta das atividades humanas, como o adoecimento dos seres humanos como resultado disto. Porém, para os autores, esta proximidade termina aí, já que a NSP tende a direcionar seu foco muito mais para as escolhas individuais e comportamentos dos cidadãos. Para a NSP, ao contrário da medicina social do século 19 , do movimento ambientalista no final do século 20, ou mesmo da saúde coletiva no Brasil, a partici pação política como principal estratégia de transformação dos problemas ambientais e de saúde tende a não ser efetivamente encorajada. 0 cidadão é transformado em um consumidor, de modo a direcionar sua atenção para longe dos problemas estruturais das sociedades industrial izadas que conformam os padrões de trabalho, produção e consumo degradantes das condições vida e que conformam injustiças ambientais, e a não desafiar o papel desempenhado pelo Estado na regulação, controle, prevenção e precaução destes problemas.

Os aspectos observados por Petersen \& Lupton (1996) no parágrafo anterior são de grande importância para se avançar não só na incorporação dos problemas ambientais na saúde coletiva, mas também na sua incorporação pelas ciências sociais, especialmente no campo da saúde. É importante notar que, no campo da saúde, tem ocorrido nas duas últimas uma identificação dos problemas ambientais com o movimento de promoção da saúde, que emergecomo uma das estratégias de uma NSP. Essa identificação é explicitada tanto na Agenda 21, em que a saúde ambiental aparece como prioridade social para a promoção da saúde, como institucionalização, no M inistério da Saúde, da gestão do sistema nacional de vigilância ambiental, que tem como um dos seus objetivos conhecer $\mathrm{e}$ estimular a interação entre saúde, meio ambiente e desenvolvimento visando ao fortalecimento da participação da população na promoção da saúde equalidade de vida (decreto 3.450, 10 de maio de 2000).

Na perspectiva da saúde coletiva, para a qual os problemas de saúde da população resultam da forma como se organiza a sociedade, em suas dimensões política, econômica e cultural, propondo então mudanças em direção tanto à democratização da sociedade, como das práticas de saúde (Paim, 2001), os programas de promoção da saúde relacionados com os problemas ambientais devem ser movimentos politicamente agressivos na perspectiva de uma eqüidade social, política e econômica (W altner-Towes, 2000). Incorporando a perspectiva das ciências sociais apontada por Vieira (1995), podemos considerar que estes programas de promoção da saúde devem ser movimentos de resoluções dos problemas ambientais de formas socialmente justas, economicamente viáveis, ecologicamente prudentes e politicamente emancipadoras. Como observa W altner-Towes (2000), o modo como os problemas são solucionados (de modo democrático e participativo, em oposição ao modo não democrático e baseado em especialistas) é tão importante como a solução encontrada, uma vez que processos e resultados possuem, ainda que separados, profundos efeitos sobre a saúde humana.

De acordo com Leff (2000), a resolução dos problemas ambientais implica a ativação e objetivação de um conjunto de processos sociais os quais as ciências sociais têm um importante papel a desempenhar. Leff (2000) destaca uma série de processos que podem se constituir em indicativos de uma agenda de pesquisa das ciências sociais em saúde sobre os problemas ambientais, sendo estes: 1) a incorporação dos valores do ambiente na ética individual, nos direitos humanos e na norma jurídica dos atores econômicos e sociais; 2) a socialização do acesso e apropriação da natureza; 3) a democratização dos processos produtivos e do poder políti$\mathrm{CO} ; 4)$ as reformas do Estado que lhe permitam mediar a resolução de conflitos de interesses em torno da propriedade e aproveitamento dos re cursos e que favoreçam a gestão participativa e descentralizada dos recursos naturais; 5) o es- 
Quadro 2

Teses e dissertações sobre a questão "ambiental" envolvendo temas e abordagens de interesse para as ciências sociais

1. M achado FBT 2000. Educação ambiental: uma experiência com grupos de adolescentes e adultos portadores de deficiência mental. Dissertação de mestrado. Faculdade de Saúde Pública, U niversidade de São Paulo. 180pp.

2. M aglio IC 2000. A descentralização da gestão ambiental no Brasil: o papel dos órgãos estaduais e as relações com o poder local 1990-1999. Dissertação de mestrado. Faculdade de Saúde Pública, Universidade de São Paulo. 270pp.

3. Roveratti DS 2000. Diagnóstico de projetos de educação ambiental em escolas públicas das áreas de proteção de mananciais da Represa Billings. Tese de doutorado. Faculdade de Saúde Pública, Universidade de São Paulo. 107pp.

4. Nazareno ER 1999. Condições de vida e saúde infantil: heterogeneidades urbanas e desi gualdades sociais em Paranaguá, Brasil. Tese de doutorado. Curso de M eio Ambiente e Desenvolvimento, Universidade Federal do Paraná. 256pp.

5. Silva ER 1998. 0 curso da água na história: simbologia, moralidade e a gestão de recursos hídricos. Tese de doutorado. Escola Nacional de Saúde Pública, Fundação O swaldo Cruz. 201pp.

6. Pelicioni AF 1998. Educação ambiental na escola: um levantamento de percepções e práticas de estudantes de primeiro grau a respeito de meio ambiente e problemas ambientais. Dissertação de mestrado. Faculdade de Saúde Pública, U niversidade de São Paulo. 118pp.

7. Toledo LM 1996. 0 espaço do cólera: determinantes sociais e regulação ambiental dos caminhos de uma epidemia. Tese de doutorado. Escola N acional de Saúde Pública, Fundação Oswaldo Cruz. 168pp.

8. Arruda M AS 1996. U ma contribuição às novas sensi bilidades com relação ao meio ambiente: representações sociais e grupos ecol ogi stas e ecofeministas cariocas. Tese de doutorado. Instituto de Psicologia, Universidade de São Paulo. 520pp.

9. Sartorato CR 1996. D ominação versus resi stência: os agrotóxicos nas lavouras dos sem-terra: representação social dos riscos à saúde dos trabalhadores. Dissertação de mestrado. Escola Nacional de Saúde Pública, Fundação O swaldo Cruz. 193pp.

10. Cunha IA 1996. Sustentabilidade e poder local: a experiência de política ambiental em São Sebastião, costa norte de São Paulo (1989-1992). Tese de doutorado. Faculdade de Saúde Pública, U niversidade de São Paulo. 527pp.

11. Toffoli FF 1996. Bom Sucesso de Itararé: o poder local e saúde em um novo micro município. Dissertação de mestrado. Faculdade de Saúde Pública, U niversidade de São Paulo. 146pp.

12. Schramm FR 1993. A terceira margem da saúde: a ética natural. Complexidade, crise e responsabilidade no saber-fazer sanitário. Tese de doutorado. Escola N acional de Saúde Pública, Fundação Oswaldo Cruz. 378pp.

13. Freitas CM 1992. A social construção de análises de riscos tecnológi cos a saúde e ao meio ambiente: estudo de uma controvérsia na arena pública. Dissertação de mestrado. Coordenação dos Programas de Pós-graduação em Engenharia, Universidade Federal do Rio de Janeiro. 217pp.

14. M arino SRA 1992. Participação popular e desenho organizacional: um estudo do Consel ho M unicipal de Saúde de Florianópolis. Dissertação de mestrado. Faculdade de Administração, U niversidadeFederal de Santa Catarina. 160pp.

15. Barani E 1990. Efeitos socioambientais decorrentes da implantação da Caraíba M etais: a percepção dos habitantes de Lamarão do Passe - Bahia. Dissertação de mestrado. Faculdade de M edicina, U niversidade Federal da Bahia. 93pp.

16. M artins IS 1985. A dimensão biológica e social da doença. Tese de livre docência. Faculdade de Saúde Pública, Universidade de São Paulo. 197pp.

17. Costa N R 1983. Estado e políticas de saúde pública (1889-1930). Dissertação de mestrado. Instituto de Pesquisas do Rio de Janeiro. 198pp.

Fonte: Literatura Latino-Americana e do Caribe em Ciências da Saúde - Biblioteca Virtual em Saúde 
Quadro 3

Artigos publicados em revistas científicas brasileiras, entre 1992 e 2001, contendo temas de interesse das ciências sociais

1. M ohr A \& Schall VT 1992. Rumos da educação em saúde no Brasil e sua relação com a educação ambiental. Cadernos de Saúde Pública 8(2):199-203.

2. Gomez CM \& Carvalho SM TM 1993. Social inequalities, labor, and health. Cadernos de Saúde Pública 9(4):498-503.

3. Schall VT 1994. Environmental and health education for school-age children: a transdisciplinary approach. Cadernos de Saúde Pública 10(2):259-263.

4. Zuñiga CG, Peña PH, Guerrero CO et al. 1994. Evaluación económica y toma de decisiones en salud ambiental. Revista de Saúde Pública 28(2):153-166.

5. Silva ER \& Schramm FR 1997. A questão ecológica: entre a ciência e a ideologia/utopia de uma época. Cadernos de Saúde Pública 13(3):355-382.

6. Porto M FS \& Freitas CM 1997. Análise de riscos tecnológicos ambientais: perspectivas para o campo da saúde do trabalhador. Cadernos de Saúde Pública 13(suppl.2):59-72.

7. Duval G 1998. Salud y ambiente en el proceso de desarollo. Ciência e Saúde Coletiva 3(2):7-16

8. Franco T \& Druck G 1998. Padrões de industrialização, riscos e meio ambiente. Ciência e Saúde Coletiva 3(2):61-72.

9. Grynszpan D 1999. Educação em saúde e educação ambiental: uma experiência integradora. Cadernos de Saúde Pública 15(suppl.2):133-138.

10. Dias JCP 2001. D oença de Chagas, ambiente, participação e Estado. Cadernos de Saúde Pública 17(suplemento):165-169.

Fonte: Ciência e Saúde Coletiva, Cadernos de Saúde Pública e Revista de Saúde Pública

tabelecimento de uma legislação ambiental eficaz que normatize os agentes econômicos, o governo e a sociedade civil; 6) as transformações institucionais que permitam uma administração transetorial do desenvolvimento; 7) a reorientação interdisciplinar do desenvolvimento do conhecimento e da formação profissional dos profissionais no campo da saúde coletiva. Todos esses processos implicam a necessidade de se avançar na reflexão sobre a pesquisa das ciências sociais no campo dos problemas ambientais que afetam a saúde coletiva.

A superação do realismo ambiental e do biologismo dominante na saúde, da naturalização das injustiças e desigualdades da vida social e da submissão ao modelo hegemônico das ciências naturais e das engenharias na compreensão e solução dos problemas ambientais representam desafios importantes para que as ciências sociais contribuam para que possamos avançar no marco conceitual da saúde coletiva. Até mesmo porque, como observa H erculano (2000) em seu artigo sobre as origens, enfoques metodológicos e objetos da sociologia ambiental, a convergência das ciências sociais ( $a$ autora centra sua análise somente na sociologia) com a saúde coletiva talvez seja uma das contribuições mais interessantes à compreensão dos problemas ambientais e uma das áreas mais necessárias a realização de pesquisas. 


\section{Referências bibliográficas}

Andler D 1987. Problème: une clé universelle?, pp. 119158. In I Stengers. D'une sience a I'autre - des concepts nomades. Centre National des Letres, Paris.

Beck U 1997. A reinvenção da política: rumo a uma teoria da modernização reflexiva, pp. 11-71. In U Beck, A Giddens \& S Lash. M odernização reflexiva - política, tradição e estética na ordem social moderna. Editora Unesp, São Paulo.

Canesqui AM 1998. Ciências sociais e saúde no Brasil: três décadas de ensino e pesquisa. Ciência e Saúde Coletiva 3(1):131-168.

Freitas CM , Soares M \& Porto M FS 1999. Subsídios para um programa na Fiocruz sobre saúde e ambiente no processo de desenvolvimento, pp. 1-11. I Seminário Saúde e Ambiente no Processo de Desenvolvimento, 2 a 5 de junho de 1998: 0 engajamento da Fiocruz. Fiocruz, Rio de Janeiro. (Série Fiocruz - Eventos Científicos)

Giddens A 1990. As conseqüências da modernidade. Editora Unesp, São Paulo.

Gochfeld M \& Goldstein BD 1999. Lessons in environmental health in the twentieth century. Annual Review of Public Health 20:35-53.

Herculano SC 2000. Sociologia ambiental: origens, enfoques metodológicos e objetos. M undo \& vida: alternativas em estudos ambientais 1(1/2):45-55.

Leal M C, Sabroza PC, Rodriguez RH \& Buss PM (orgs.) 1992a. Saúde, ambiente e desenvolvimento - uma análise interdisciplinar. Editora H ucitec-Abrasco, São Paulo-Rio de Janeiro, 295pp.

Leal MC, Sabroza PC, Rodriguez RH \& Buss PM (orgs.) 1992b. Saúde, ambiente e desenvolvimento - processos e conseqüências sobre as condições de vida. Editora Hucitec-Abrasco, São Paulo-Rio de Janeiro, 307pp.

Leff E 2000. Pensamento sociológico, racionalidade ambiental e transformações do conhecimento, pp. 109157. In E Leff. Epistemologia ambiental. Cortez Editora, São Paulo.

M acnaghten P \& U rry J 1998. Contested natures. Sage Publications Ltd., Londres, 307pp.
M inayo MCS et al. 1999. O programa institucional sobre saúde e ambiente no processo de desenvolvimento da Fundação Oswaldo Cruz. Anais da Academia Brasileira de Ciências 71(2):279-288.

M inayo M CS 1997. Pós-graduação em saúde coletiva: um projeto em construção. Ciência e Saúde Coletiva 2(1/2):53-71.

Paim JS 2001. M odelos assistenciais: reformulando o pensamento e incorporando a proteção e a promoção da saúde. AN VISA - Seminários Temáticos Permanentes. Braślia: 28 de março de 2001.

Paim JS \& Almeida Filho N 1998. Saúde coletiva: uma "nova saúde pública" ou campo aberto a novos paradigmas? Revista de Saúde Pública 32(4):299-316.

Petersen A \& Lupton D 1996. The new public health health and self in the age of risk. Sage Publications, Londres.

Porto M FS 1998. Saúde, ambiente e desenvolvimento: re flexões sobre a experiência da COPASAD - Conferência Pan-Americana de Saúde e Ambiente no Contexto do Desenvolvimento Sustentável. Ciência e Saúde Coletiva 3(2):33-46

Samajaj 2000. A reprodução social ea saúde. Casa da Qualidade Editora, Salvador, 103pp.

Schakley S, Wynne B \& W aterton C 1996. The past, present and future potential of complex thinking. Futures 28(3):201-225.

Vieira PF 1995. A problemática ambiental e as ciências sociais no Brasil (1980-1990), pp. 103-147. In DJ Hogan e PF Vieira (orgs.). Dilemas socioambientais e desenvolvimento sustentável. Editora da U nicamp, Campinas.

Tambellini AT \& Câmara VM 1998. A temática saúde e ambiente no processo de desenvolvimento do campo da saúde coletiva: aspectos históricos, conceituais e metodológicos. Ciência e Saúde Coletiva 3(2):47-59.

W altner-Towes $D$ 2000. The end of medicine: the beginning of health. Futures 32(7):655-667.

Artigo apresentado em 20/6/2002

Aprovado em 15/9/2002

Versão final apresentada em 16/12/2002 